\title{
APROXIMACIÓN A UN ESTUDIO DE LAS CLÁUSULAS ABUSIVAS DE LA CONTRATACIÓN EN EL NUEVO ESTATUTO DEL CONSUMIDOR
}

\section{Fernando Jiménez Valderrama}

Doctor en Derecho por la Universidad de Salamanca (España). Máster en Derecho de la Unión Europea por la Universidad Carlos III de Madrid (España). Abogado de la Universidad del Rosario. Profesor en las Universidades San Pablo - CEU, Complutense y Carlos III de Madrid y Director del Instituto Europeo de Estudios Maritimos.

Es miembro del Colegio de Abogados Comercialistas, la Asociación Española de Derecho Marítimo y del Ilustre Colegio de Abogados de Madrid (España). Profesor y Jefe del Área de Derecho Privado y de la Empresa de la Facultad de Derecho de la Universidad de La Sabana.

Correo electrónico:ferjiva@gmail.com

\begin{abstract}
Resumen
El nuevo Estatuto del Consumidor mediante la Ley 1480 de 2011 ha puesto en primera plana un necesario debate acerca de la actual situación de las relaciones de consumo y su regulación según los últimos instrumentos diseñados en el ámbito jurídico mercantil. En este escrito se expone la necesidad de comprender la importancia práctica de cualquier normativa destinada a regular en una gran mayoría las operaciones mercantiles que diariamente se realizan en el marco del desarrollo de la empresa y su actividad comercial. Palabras Clave: Cláusulas abusivas, estatuto del consumidor, relaciones de consumo.
\end{abstract}

\begin{abstract}
The new Statute of the Consumer by means of the Law 1480 of 2011 has put in the first page a necessary debate brings over of the current situation of the relations of consumption and his regulation according to the last instruments designed in the juridical mercantile area. In this writing there is exposed the need to understand the practical importance of any regulation destined to regulate in a great majority the mercantile operations that every day are realized in the frame of the development of the company and his commercial activity.
\end{abstract}

Keywords: Improper clauses, statute of the consumer, relations of consumption.

\section{Résumé}

Le nouveau Statut du Consommateur au moyen de la Loi 1480 de 2011 a mis à une première taloche de maçon un débat nécessaire à propos de l'actuelle situation des relations de consommation et sa régulation selon les derniers instruments dessinés dans le domaine juridique mercantile. Dans cet écrit s'expose la nécessité de comprendre l'importance pratique de n'importe quelle réglementation destinée à régler dans une grande majorité les opérations mercantiles qui sont quotidiennement réalisées dans le cadre du développement de l'entreprise et son activité commerciale.

Mots-clés: Des clauses abusives, un statut du consommateur, des relations de consommation. 

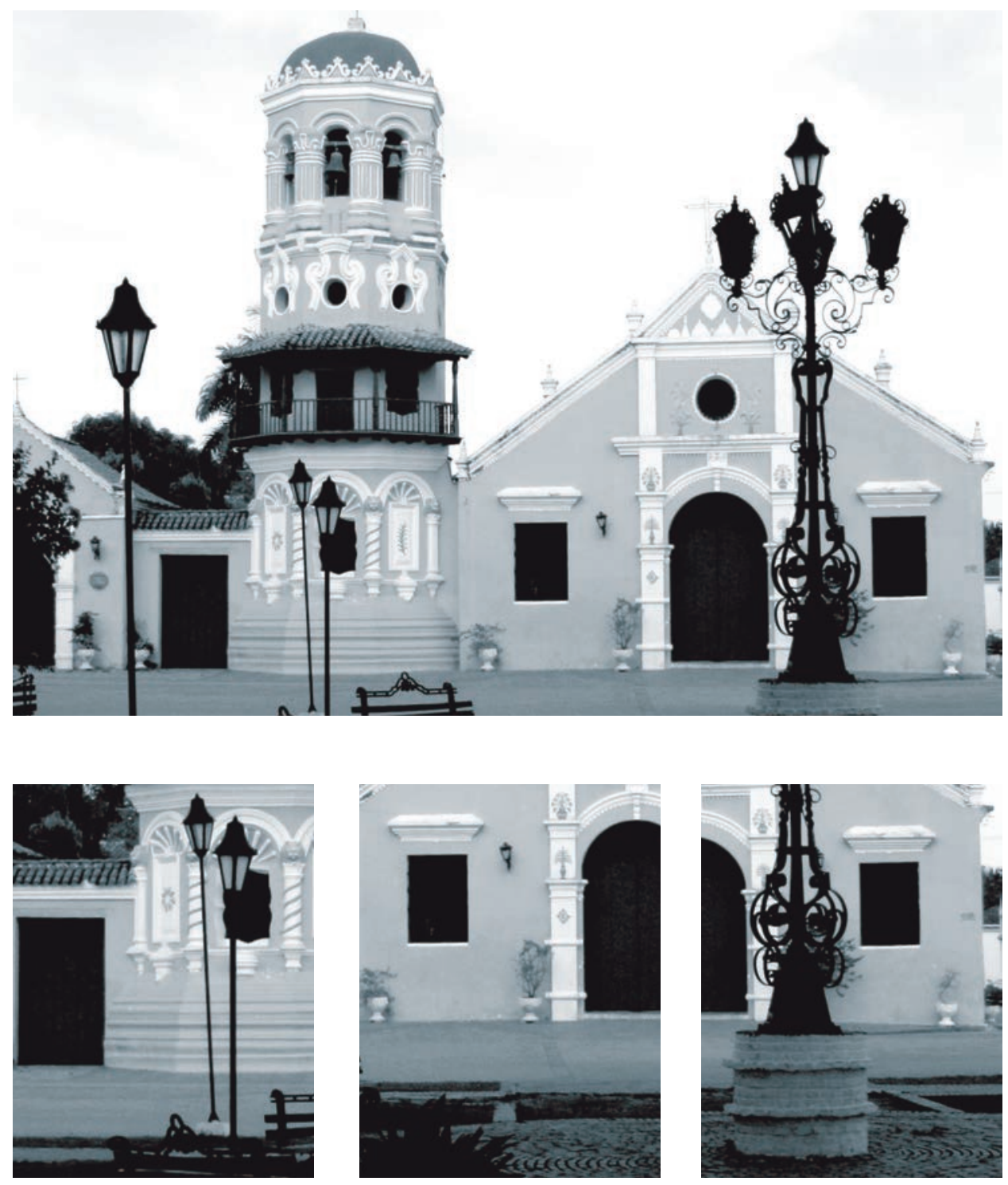

Iglesia de Santa Bárbara - Mompóx - Colombia Martín Emilio Hernández Manrique 


\section{APROXIMACIÓN A UN ESTUDIO DE LAS CLÁUSULAS ABUSIVAS DE LA CONTRATACIÓN EN EL NUEVO ESTATUTO DEL CONSUMIDOR*}

Fernando Jiménez Valderrama

La aprobación del nuevo Estatuto del Consumidor mediante la Ley 1480 de 2011 ha puesto en primera plana un necesario debate acerca de la actual situación de las relaciones de consumo y su regulación según los últimos instrumentos diseñados en el ámbito jurídico mercantil.

Como en diferentes foros lo ha expresado el Prof. Álvaro Mendoza Ramírez, el Derecho del Consumidor ha asumido un especial protagonismo en el mundo moderno a tal punto de convertirse en uno de los pilares de la regulación mercantil, pues desplaza otros ámbitos normativos que paulatinamente han tenido un papel cada vez más residual. Indudablemente que el volumen de operaciones mercantiles que se realizan a partir del hecho del consumo superan de forma amplia no solo en número sino en valor las operaciones mercantiles que podríamos ahora llamar tradicionales.

Estas afirmaciones nos permiten comprender la importancia práctica de cualquier normativa destinada a regular en una gran mayoría las operaciones mercantiles que diariamente se realizan en el marco del desarrollo de la empresa y su actividad comercial.

En esta materia tradicionalmente la doctrina y la jurisprudencia han coincidido en un punto de partida: la base conceptual de todo el andamiaje jurídico de regulación del consumo surge de la necesidad de proteger a la parte débil en la contratación mercantil. Si bien en los momentos originarios del derecho mercantil

\footnotetext{
El presente escrito se deriva del proyecto: Transformaciones del ordenamiento jurídico colombiano a partir de la vigencia de los tratados de libre comercio con Estados Unidos y la Unión Europea: El transporte marítimo, la propiedad industrial, la contratación pública, la actividad financiera y aseguradora, y los derechos laborales de los trabajadores. Universidad de la Sabana. Convocatoria Interna 2011 - 2012. Universidad de la Sabana. Bogotá.
} 
los esquemas jurídicos de derecho civil, adaptados a las nuevas necesidades de los comerciantes resultaban suficientes para regular las operaciones mercantiles, pronto ello empezaría a cambiar por las nuevas necesidades que exigían la evolución de un sistema económico cada vez más complejo.

En el ámbito contractual, la defensa a ultranza del principio de autonomía de la voluntad privada como mecanismo generador de obligaciones y contratos, pronto empezaría a ser cuestionado ante una realidad incontestable: la diferencia de poder de negociación entre la gran empresa y el destinatario final de los productos o servicios, entendido como la parte débil en esta relación negocial. Indudablemente que esta diferencia de poder de negociación empezó a traducirse en la realidad de la negociación en una abierta imposición de las condiciones contractuales que podrían incluso llegar a cuestionar la real existencia de una auténtico consentimiento, base fundamental para la creación de obligaciones y contratos.

Esta diferencia en poder de negociación se hizo patente en múltiples sectores de la actividad comercial. A manera ejemplificativa se puede mencionar que en el ámbito del transporte marítimo bajo conocimiento de embarque desde muy temprano se empezaron a presentar situaciones claramente abusivas en contra de los cargadores de las mercancías que veían impuestas estipulaciones contractuales que rompían los mecanismos tradicionales de asignación de responsabilidad, al trasladar la gran mayoría de los riesgos de la operación a la parte más débil en la contratación.

Esta situación que se presentaba en el transporte marítimo también ocurría en otros sectores de la actividad comercial primordialmente donde actúa la gran empresa.

Ahora bien, si bien el propio ordenamiento jurídico privado ya contaba con mecanismos como el principio de buena fe contractual y la regulación de contratos de adhesión que permitían equilibrar situaciones claramente abusivas en la contratación, estas figuras resultaban aún insuficientes para proteger los intereses de la parte débil en un ámbito de negociaciones generalizadas.

Era por lo tanto imperativo, en términos de justicia y para proteger la competencia y el desarrollo del mercado, el establecimiento de una normativa detallada y completa que amparase a la parte débil en la contratación mercantil. A partir de esta idea se empiezan a configurar las primeras instituciones jurídicas que regulan la relación de consumo con la finalidad de establecer un mecanismo de protección al consumidor, considerado como la parte débil en esta relación.

Sin embargo, el establecimiento de una normativa particular propia para las relaciones de consumo no implica y ello es bueno resaltarlo, que hayan perdido vigencia los mecanismos que podríamos llamar tradicionales basados, entre otros principios, en la buena fe y la protección a la parte débil en los contratos de adhesión, cuya aplicación aún está vigente para todos los contratos e inspira también las normas particulares en materia de contratos donde intervienen consumidores.

Obviamente que la nueva regulación protectora de la posición del consumidor frente a los eventuales abusos que puede ser parte frente a empresarios o grupos de empresarios, desde muy temprano manifestó una abierta colisión con los principios 
que tradicionalmente habían regulado las operaciones mercantiles y que resultaban nucleares en el ámbito jurídico privado. Me refiero a principios como el de libertad de contratación y el principio de seguridad contractual, la relatividad del negocio jurídico y la igualdad de las partes ante la ley.

Precisamente la necesidad de respetar estos principios haría que la nueva estructura del derecho del consumo gravitara entre la indispensable protección que se debe dar a los consumidores y la observancia a los principios tradicionales antes mencionados.

Desde nuestro punto de vista esta colisión debe resolverse de manera equilibrada sin caer en posiciones extremas. Una intervención ilimitada por parte del legislador en la voluntad de los contratantes, a tal punto de suplirla en su totalidad so pretexto de amparar a la parte más débil en la contratación, no resultaría conveniente además de que no sería aceptable por los operadores económicos tanto locales, como de manera principal, internacionales, cuyas negociaciones se basan al menos en el momento actual en el respeto al principio de libertad de contratación. Por el contrario, un respeto absoluto por el principio de disposición entre las partes y de libertad de contratación, entre otros, conlleva, como se ha podido comprobar, a que se presenten situaciones claramente abusivas en contra de la parte débil de la contratación.

Son múltiples los puntos que podrían examinarse, en esta materia, sin embargo, por razón de la temática que nos ha sido designada, nos centraremos en la protección del consumidor frente a cláusulas abusivas en la contratación.

El primer punto de análisis se refiera a los tipos contractuales que podrían contener cláusulas abusivas. Si partimos de la base de la necesidad de una especial vigilancia y eventual corrección de los pactos establecidos cuando se presentan situaciones de abuso causado por el desequilibrio de poderes de negociación entre las partes, parecería que sólo "los contratos de adhesión” podrían contener este tipo de cláusulas.

Sin embargo, tal como hemos mencionado, el legislador ha preferido configurar un sistema autónomo distinto de los mecanismos de corrección tradicionales de derecho privado, entre ellos la especial regulación de los contratos de adhesión. Para configurar este nuevo esquema se han trazado unas nuevas líneas de base sobre el concepto de "relación de consumo" a partir de las cuales ha podido estructurar un andamiaje completo y suficiente adaptado a las nuevas necesidades del mercado.

En el caso particular colombiano, el artículo 2 del Estatuto del Consumidor afirma que:

"las normas de esta ley regulan los derechos y obligaciones surgidas de los productores, proveedores y consumidores y la responsabilidad de los productores y proveedores, tanto sustancial como procesalmente. Las normas contenidas en esta ley son aplicables en general a las relaciones de consumo y a la responsabilidad de los productores y proveedores frente al consumidor en todos los sectores de la economía respecto de los cuales no exista regulación especial (...)”. 
Se opta, como hemos insinuado, por establecer un amplísimo espectro de aplicación de protección basado en la denominada "relación de consumo", dejando a un lado consideraciones relativas a un(os) determinado(s) tipos de contratos, particularmente los contratos de adhesión.

Igualmente debemos destacar que, tal como viene regulado en el nuevo Estatuto, la relación de consumo puede realizarse en cualquier sector económico, aunque este criterio expansivo se ve peligrosamente limitado por el parágrafo 2 del artículo 2 de la Ley que establece que el Estatuto se aplicará a todas las relaciones de consumo “respecto de los cuales no exista una regulación especial".

Decimos desafortunada, porque ello puede conllevar a que un importante grupo de consumidores se vean desposeídos en claro fraude de ley de sus derechos por la vía de "regulaciones especiales" que dicte el gobierno, previsiblemente con menor nivel de protección y con eventuales consecuencias discriminatoria frente a otros consumidores.

Una vez establecido el marco de contratos en los que podemos encontrar "cláusulas abusivas", es necesario detenernos aunque brevemente en el concepto de “consumidor”. En los términos del Estatuto (artículo 5.3) consumidor es

"toda persona natural o jurídica que, como destinatario final, adquiera o utilice un determinado producto, cualquiera que sea su naturaleza para la satisfacción de una necesidad propia, privada, familiar o doméstica y empresarial cuando no esté ligada intrínsecamente a su actividad económica. Se entenderá incluido en el concepto de consumidor el de usuario".

En este punto simplemente es conveniente reiterar lo que doctrinalmente ya es generalmente aceptado respecto a la definitiva inserción de las personas jurídicas como "consumidores" en la medida que sean destinatarias finales de productos o servicios y en cuanto la adquisición del producto o la realización del servicio no esté ligada "intrínsecamente" a su actividad económica empresarial.

De esta manera las relaciones jurídicas tanto contractuales como precontractuales y posteriores a la firma del contrato que establezcan personas naturales o jurídicas y que se puedan catalogar como de consumo son finalmente reguladas por el Estatuto y susceptibles de integrar "cláusulas abusivas" que afecten los intereses de los consumidores.

Una vez examinados estos puntos preliminares, corresponde, para seguir la guía establecida para esta conferencia, tratar particularmente las cláusulas abusivas que pueden insertarse en contratos que afectan los intereses de los consumidores.

La protección del consumidor frente a estas cláusulas aparece regulada en el Capítulo III del nuevo Estatuto (Cláusulas Abusivas) del Título VII (Protección precontractual).

El ámbito de las cláusulas abusivas en la contratación, como lo hemos insinuado, es uno de esos ámbitos en los cuales se evidencia abiertamente el conflicto entre el interés de proteger al consumidor y el reconocimiento de los principios de seguridad contractual, de libertad de contratación, de relatividad del negocio jurídico y de igualdad de las partes ante la ley. 
El desequilibrio en poder de negociación que, por ejemplo, se presentaba en sectores desarrollados por la gran empresa, de hecho generaban la inclusión de cláusulas en los contratos a través de los cuales se mermaba considerablemente los derechos de la parte débil e implicaba una nueva distribución de riesgos en la ejecución del contrato a favor de una de las partes.

Indudablemente que esta situación podía, incluso, llegar a negar la existencia misma del contrato, en la medida que cuestionaba claramente la expresión de un consentimiento real y libre por parte de uno de los contratantes.

Como reacción a esta situación los ordenamientos jurídicos contemporáneos han diseñado toda una estructura jurídica que limite esa libertad de pactos al intervenir abiertamente a favor de la parte débil de la relación de consumo.

Tradicionalmente han existido distintos mecanismos de limitación de la voluntad contractual en este ámbito. En el primer grupo podemos encontrar los llamados mecanismos de "auto-regulación" en los cuales son los propios empresarios quienes a través de asociaciones y mediante distintos mecanismos como los códigos éticos, deciden de forma autónoma establecer reglas que impidan la imposición de cláusulas claramente abusivas en la contratación. Estos instrumentos si bien deben reconocerse como elementos importantes en el control de situaciones abusivas en la contratación, han demostrado ser insuficientes.

Ante esta situación, el Estado ha intervenido legislativamente para establecer otros mecanismos de protección del consumidor y corrección del sistema, primordialmente articulados a través de la actuación administrativa de entidades públicas y la intervención de los Jueces. Estos instrumentos también son susceptibles de aplicarse de manera conjunta y coordinada para obtener los mejores resultados en el cumplimiento de esta política.

Parte de esta intervención estatal es la normativa que se analiza en defensa de los derechos de los consumidores, así como todo el cuerpo administrativo y judicial preparado para hacer el seguimiento y control correspondiente en aplicación de esta regulación especial.

El primer punto de aplicación de los instrumentos antes mencionados es la determinación de los criterios para establecer cuándo una cláusula contractual es "abusiva" y, por lo tanto, objeto de reproche jurídico, bien sea para declarar su nulidad, ineficacia o bien para mantener su validez y eficacia pero aplicados los correctivos necesarios para hacerla acorde con el ordenamiento jurídico.

Tanto en el sistema de intervención administrativa como en el judicial se han diseñado distintos criterios para establecer la abusividad de los pactos realizados entre particulares. Así podemos mencionar, en primer lugar, el sistema de tarifa legal, en el cual por vía principalmente legal se establece un listado de cláusulas que se consideran siempre abusivas, se ordena a los jueces declarar o reconocer su ineficacia de forma automática.

El artículo 43 del Estatuto del Consumidor establece que "son ineficaces de pleno derecho las cláusulas que (...)" y a continuación menciona 14 supuestos de pactos 
contractuales que se consideran per se abusivos y, por lo tanto, ineficaces a la luz de nuestro ordenamiento jurídico.

Si bien este sistema otorga claridad a los operadores económicos, no deja de ser incompleto en la medida que pueden existir pactos abusivos que no figuren en el listado o, por el contrario, pactos que se realicen entre contratantes con poder de negociación similar o incluso diferenciado, pero sobre los que claramente se haya expresando una voluntad plenamente informada y libre y sobre los cuales en mi criterio no debería haber ningún juicio de reproche por parte del ordenamiento jurídico.

El sistema de tarifa legal, denominado también de listas negras, amarra al Juez frente a los supuestos legales impidiéndole entrar a analizar el hecho mismo de la negociación del contrato y, por lo tanto, determinar cuándo realmente se presenta una situación de abuso en la celebración del mismo.

Frente al sistema de tarifa legal o de listas negras algunos sectores de la doctrina han propugnado por un sistema basado en el establecimiento de una cláusula general, que pueda ser utilizada para regular cualquier contrato basado en una relación de consumo y para realizar el correspondiente juicio de abusividad de su clausulado.

Como criterio general estos mecanismos acuden a conceptos como el principio de buena fe normalmente completado con criterios objetivos como la comprobación de un perjuicio o detrimento que se cause al consumidor por la presencia de una cláusula abusiva en el contrato. En términos del artículo 3 de la Directiva Comunitaria Europea 1993/13/CEE, de 5 de abril, sobre cláusulas abusivas en los contratos celebrados con consumidores, una cláusula es abusiva cuando, "pese a las exigencias de la buena fe, causa un detrimento del consumidor, un desequilibrio importante entre los derechos y obligaciones de las partes que se derivan del contrato”. La Ley española 7/1998 de 13 de abril, de transposición de la mencionada norma comunitaria, establece en el artículo 10 bis que se consideran abusivas "todas aquellas estipulaciones no negociadas individualmente que en contra de las exigencias de la buena fe causen, en perjuicio del consumidor, un desequilibrio de lo importante de los derechos y obligaciones de las partes que se deriven del contrato”. A su vez el artículo 42 del Estatuto del Consumidor colombiano califica como abusivas aquellas cláusulas

"que producen un desequilibrio injustificado en perjuicio del consumidor y las que, en las mismas condiciones, afecten el tiempo, modo o lugar en que el consumidor puede ejercer sus derechos. Para establecer la naturaleza $y$ magnitud del desequilibrio, serán relevantes todas las condiciones particulares de la transacción particular que se analiza".

A partir de las normas antes mencionadas podemos hacer algunos comentarios preliminares: tanto el sistema comunitario europeo como el colombiano han incorporado en su regulación el sistema de cláusula general. En el sistema comunitario europeo se incorpora el criterio de la "buena fe" como elemento en el examen de abusividad de la cláusula, a diferencia del sistema colombiano que no lo incorpora 
expresamente, aunque en mi criterio es aplicable en la medida que la buena fe es criterio general de regulación de la formación, celebración y ejecución de todos los contratos, no solamente aquellos que implican una relación de consumo.

En ambos ordenamientos el examen de abusividad puede realizarse respecto de cláusulas pactadas en cualquier tipo de contrato realizado en virtud de relaciones de consumo bajo un análisis cualitativo de todas las prestaciones que nacen en el mismo. Esta revisión cualitativa incluye en primer lugar examinar si se ha presentado un desequilibrio "importante" en el contrato (nuestro Estatuto menciona "injustificado") además de la causación de un perjuicio al consumidor en referencia a la disminución o negación de los derechos del consumidor, el aumento de sus obligaciones contractuales o bien una marcada distribución en su contra de los riesgos derivados del contrato.

Ambas normativas establecen que el desequilibrio contractual debe examinarse teniendo en cuenta el conjunto de derechos y obligaciones que surgen del contrato y las circunstancias en las cuales fue celebrado.

Igualmente, en mi criterio, la revisión cualitativa de las prestaciones excluye de hecho el examen cuantitativo del contrato, especialmente en materia de fijación de precios, cuya determinación tiene otros mecanismos de corrección como la lesión enorme, con las amplias limitaciones que tiene su aplicación en nuestro sistema jurídico exclusivamente aplicable a la compraventa de inmuebles y en las particiones y exigiendo un desequilibrio sustancial en el precio que impide someter a su regulación un importante número de eventos en los cuales es patente una inequidad. En todos los contratos, incluidos los que implican relaciones de consumo, existen unos elementos mínimos de expresión de la voluntad de las partes que dan origen al mismo, uno de ellos es la manifestación misma de la voluntad de contratar ante la necesidad de un producto o de un servicio y otro el precio que el comprador está dispuesto a pagar, éste último regulado por la ley de la oferta y la demanda en una economía de mercado. Sobre estos acuerdos y prestaciones necesarias y esenciales para la creación del contrato no debe, en mi concepto, entrar la protección sobre cláusulas abusivas, que si resultaría pertinente aplicar a las demás prestaciones destinadas a realizarse durante la ejecución del contrato y cuyo pacto abusivo puede resultar claramente lesivo y perjudicial a la parte débil en la contratación.

Finalmente quisiera hacer una reflexión crítica al sistema establecido en el Estatuto en esta materia.

En mi criterio la regulación establecida toma diversos elementos de los sistemas mencionados para configurar un sistema que podríamos llamar mixto. Así, a pesar que en el artículo 42 del Estatuto se establece una cláusula general, a continuación, en el artículo 43, se menciona un listado de cláusulas que en los términos de la ley son "ineficaces de pleno derecho", frente a las cuales al Juez no le queda otra alternativa que declarar o reconocer la ineficacia sin mayor valoración.

El establecimiento de una cláusula general permite, sin embargo, modular el sistema de cláusulas prohibidas al permitir que pueda haber otras susceptibles también 
ser declaradas ineficaces por los jueces en la medida que "produzcan un desequilibrio injustificado en perjuicio del consumidor (...)" o que "afecten el tiempo, modo o lugar en que el consumidor puede ejercer sus derechos". Sin duda alguna que en este análisis puede resultar de utilidad el listado de cláusulas prohibidas establecido en el artículo 43, en cuando ejemplificativo de estipulaciones sancionables.

Se logra de esta manera un sistema amplio que permitirá a los jueces valorar los contratos con consumidores a fin de descifrar posibles estipulaciones abusivas en su contra. Ello habla a favor del sistema aun cuando debemos reconocer que la inserción de un sistema de tarifa legal o listas negras, se traduce en una rigidez innecesaria, impidiendo supuestos en los cuales las partes voluntariamente y en ejercicio de su libertad deciden pactar estipulaciones que impliquen renuncia a derechos, asunción de obligaciones y riesgos y sobre los cuales no debería haber sanción alguna. Es al Juez a quien le correspondería considerar estos casos y si entiende que no se presentó abuso alguno y proceder, por ende, dar eficacia plena a lo pactado.

El sistema comunitario europeo y el español siguen esta senda al igual que el francés que en el artículo L 132-1 del Code de la Consommation establece:

"Dans les contrats conclus entre professionnels et non-professionnels ou consommateurs, sont abusives les clauses qui ont pour objet ou pour effet de créer, au détriment du non-professionnel ou du consommateur, un déséquilibre significatif entre les droits et obligations des parties au contrat. Un décret en Conseil d'Etat, pris après avis de la commission instituée à l'article L. 534-1, détermine une liste de clauses présumées abusives; en cas de litige concernant un contrat comportant une telle clause, le professionnel doit apporter la preuve du caractère non abusif de la clause litigieuse".

Ello a pesar de que, posteriormente, en el mismo artículo el legislador francés borra con el codo lo que escribe con la mano o al menos genera confusión al afirmar:

"Un décret pris dans les mêmes conditions détermine des types de clauses qui, eu égard à la gravitédes atteintes qu'elles portent à l'équilibre du contrat, doivent être regardées, de manière irréfragable, comme abusives au sens du premier alinéa".

\title{
REFERENCIAS
}

\author{
ÁLVAREZ GONZÁLEZ, Santiago: Condiciones Generales en la Contratación \\ Internacional. Madrid, Editorial La Ley, 1992, 187 p. \\ ESTEBAN DE LA ROSA, F.: Régimen jurídico de la contratación electrónica de \\ consumo el sistema español de Derecho Internacional Privado. En: Aranzadi \\ Civil-Mercantil, número 2/2009 (Estudio). \\ GALGANO, Francesco: El negocio jurídico. Valencia, Ed. Tirant lo Blanch, 1992, \\ $569 \mathrm{p}$.
}


GONDRA, José María: Condiciones de la contratación y protección de la parte "más débil" en el marco del Derecho uniforme de la compraventa internacional. En: Estudios de Derecho Mercantil en homenaje al Rodrigo Uría. Madrid, Editorial Cívitas, 1978, pp. 231 a 247.

LARROUMET, Christian: La protección de los consumidores contra las cláusulas abusivas estipuladas en los contratos en Derecho Comunitario europea y en Derecho Francés. En: Política y Derecho del Consumo. Bogotá, El Navegante Editores, 1998, pp. 167 a 201.

LARROUMET, Christian: Teoría General del Contrato. Bogotá, Temis, Tomos I y II, 1999.

PÉREZ CONESA, Carmen: Nulidad de la "cláusula suelo" en los contratos de préstamo hipotecario a interés variable por abusiva. En: Aranzadi CivilMercantil, número 2/2011 (Comentario).

PERTÍÑEZ VILCHEZ, Francisco: Los elementos esenciales del contrato y el control de condiciones generales. En: Aranzadi Civil-Mercantil, número 17/2003 (Estudio).

PERTÍÑEZ VÍLCHEZ, Francisco: Reflexiones sobre el carácter abusivo de la cláusula suelo en los contratos de préstamo hipotecario. En: Aranzadi CivilMercantil, número 1/2011 (Estudio).

REGLERO CAMPOS, L. Fernando: Régimen de ineficacia de las condiciones generales de la contratación. Cláusulas no incorporadas y cláusulas abusivas: concepto y tipología, en: Aranzadi Civil-Mercantil, número 17/2003 (Estudio).

RUBIO TORRANO, Enrique: Hacia la mejora en la protección de los consumidores y usuarios. En: Aranzadi Civil-Mercantil, número 5/2006 (Tribuna). 



\title{
INMIGRANTES Y EMIGRANTES Barrancabermeja territorio de contrastes históricos
}

\author{
Mario Andrés Alvarado Lozano \\ Especialista en Derecho Penal UNAB. Especialista en Ciencias Políticas UNAB. Psicólogo UNAB. Abogado. \\ Universidad Autónoma de Bucaramanga UNAB. Profesional en Estudios Literarios UNAB. Director del Grupo \\ de Investigaciones Facultad de Derecho, Sede Barrancabermeja-Estudios Socio-jurídicos. Docente Investigador \\ Universidad Cooperativa de Colombia UCC, Sede Barrancabermeja (Colombia). Facultad de Derecho. \\ Correo electrónico: mario.alvarado@campusucc.edu.co; alvaradoma001@hotmail.com
}

\section{Resumen}

El proyecto de investigación desarrollado tiene como objetivo central analizar la problemática socio-jurídica, familiar y personal que con mayor frecuencia presenta la población usuaria del programa de "Asistencia Jurídica Integral a Población Desplazada". Para lograr dicho objetivo la investigación se asume un diseño denominado triangulación metodológica o aproximación por métodos múltiples, dentro del cual se utilizan instrumentos de recolección de información cuantitativa como la encuesta y cuestionarios, y cualitativos como la entrevista, grupos focales y pruebas psico-diagnósticas proyectivas o subjetivas. Se espera que los resultados de la investigación arrojen luces en relación con el impacto directo que tiene sobre el proyecto de vida individual y colectiva de las personas la violación de los derechos humanos y el sometimiento a experiencias traumáticas asociadas con la vivencia del desplazamiento forzado.

Palabras Clave: Desplazamiento forzado, caracterización, violencia, conflicto armado, exclusión, educación.

\begin{abstract}
The research project aims to analyze the central socio-legal issues, family and personal that most often presents the users of the program "Legal Assistance to force displacement Integral". To achieve this research assumes a design called methodological triangulation or multiple methods approach, within which used data collection instruments and quantitative and survey questionnaires and qualitative interviews, focus groups and projective psychodiagnostic tests or subjective. It is expected that the results of the research will shed light on the direct impact on the draft individual and collective lives of people the human rights violation and submission to traumatic experiences associated with the experience of forced displacement.
\end{abstract}

Keywords: Forced displacement, characterization, violence, armed conflict, exclusion, education.

\section{Résumé}

Le projet de recherche développé a pour un objectif central analyser la problématique un partenaire juridique, familiale et personnel qu'avec une plus grande fréquence présente la population utilisatrice du programme de "Aide Juridique Intégrale à la Population Déplacée. Pour obtenir le dit objectif la recherche assume un dessin dénommé une triangulation méthodologique ou approche par des méthodes multiples, à l'intérieur qui sont utilisés des instruments de récolte d'information quantitative comme l'enquête et les questionnaires, et qualitatifs comme l'interview, les groupes focaux et tu essaies psicodiagnósticas proyectivas ou subjectives. Il s'attend que les résultats de la recherche lancent des lumières en relation avec l'impact direct qui a sur le projet de vie individuelle et collective des personnes la violation des droits de l'homme et de la soumission aux expériences traumatiques associées à l'expérience du déplacement forcé.

Mots-clés: le Déplacement forcé, la caractérisation, la violence, le conflit armé, l'exclusion, l'éducation. 

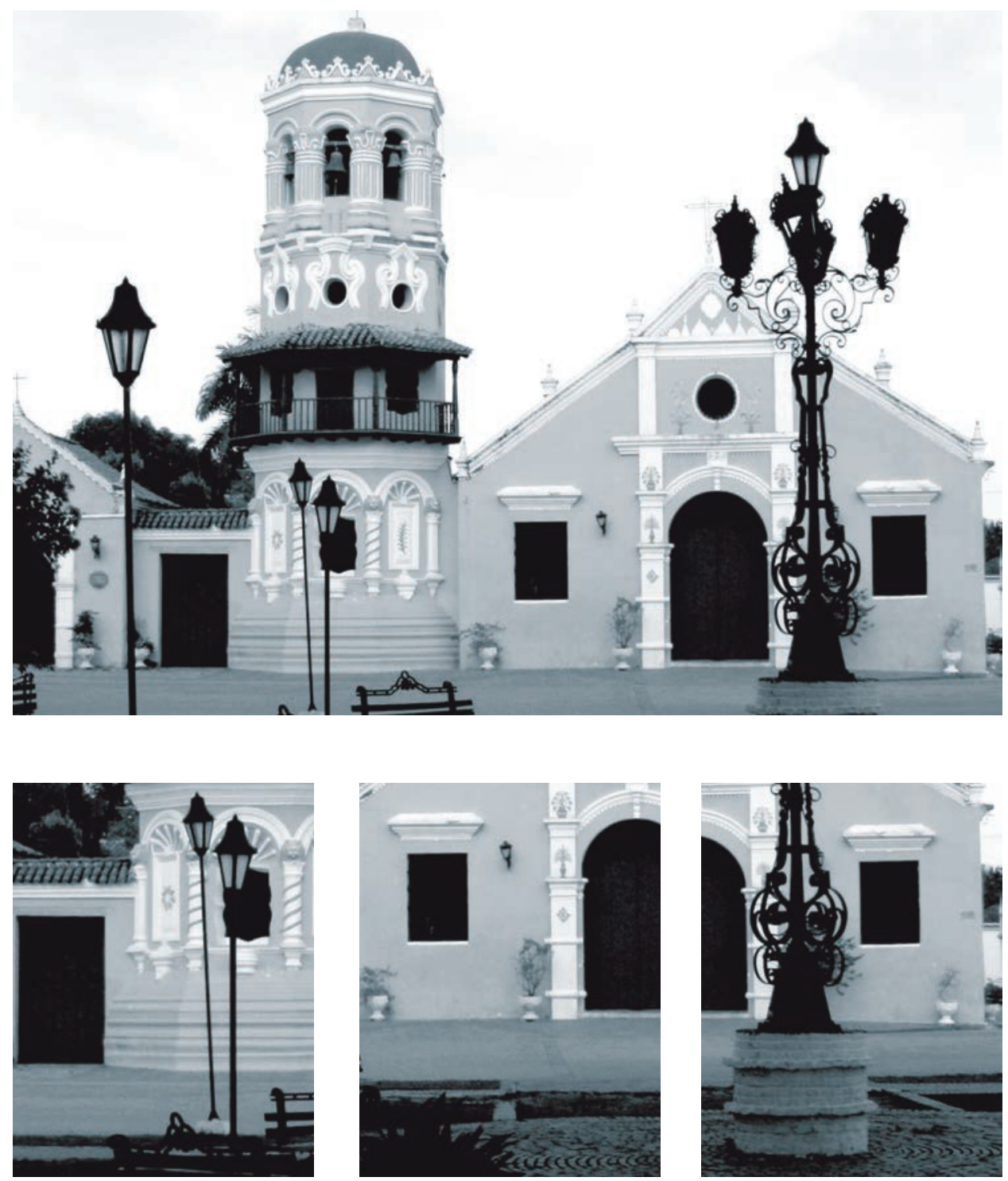

Iglesia de Santa Bárbara - Mompóx - Colombia

Martín Emilio Hernández Manrique 


\section{INMIGRANTES Y EMIGRANTES Barrancabermeja territorio de contrastes históricos*}

Mario Andrés Alvarado Lozano

Barrancabermeja existe como municipio desde 1922, sin embargo, su historia se remonta a la época precolombina; antes de la llegada de los españoles existía en este territorio la comunidad aborigen Yariguíes, quienes opusieron resistencia a los procesos de colonización implementados por los europeos. La existencia de abundante material vegetal (Caucho, Palma de Tagua, y Quina) de uso importante durante el siglo XVIII y XIX, motivó la llegada de extraños a los territorios de Barrancabermeja. Posteriormente el descubrimiento de petróleo y con ello la instalación de la Tropical Oil Company, trajo consigo inmigrantes de diversas regiones de Colombia, que veían en este lugar, la oportunidad para obtener un empleo. Así se formó la mezcla cultural que aún persiste en nuestros días.

La economía de Barrancabermeja está constituida por varias actividades entre las que se destacan la agropecuaria y en ella la producción de cacao, maíz, plátano, yuca, cítricos y una significativa población bobina, caprina y bovina. En este aparte se observa que la distribución y uso la tierra constituye una limitante para el desarrollo agrícola del municipio, si se tiene en cuenta que el $81.4 \%$ de los propietarios sólo tienen el $17.9 \%$ de la tierra, mientras que $4.1 \%$ de propietarios tienen el $45.6 \%$ de la tierra. Este nivel de concentración de la propiedad limita la posibilidad de implementación y desarrollo de la economía campesina para autoconsumo y abastecimiento local, por lo que el municipio tiene como despensa agrícola los municipios circunvecinos San Vicente de Chucurí, Yondó y Cantagallo, entre otros. El subsector pesquero también contribuye con este renglón de la economía local, en tanto que se comercializan anualmente 768 toneladas de carne de pescado de especies nativas de la región.

\footnotetext{
* El artículo es producto de la investigación: Vulnerabilidad de Derechos de población en situación de desplazamiento interno asentada en el municipio de Barrancabermeja. - Convocatoria Nacional General Comité Nacional de Investigación CONADI (UCC), Año 2010, adelantada por el autor. Grupo de Investigación Estudios Socio jurídicos UCC Sede Barrancabermeja (Colombia).
} 
Con relación al desplazamiento forzado, históricamente Barrancabermeja ha sido un centro receptor de inmigrantes, que por una $u$ otra circunstancia llegan a la ciudad. El documento del PIU, identifica tres causas de recepción a saber:

a. La economía petrolera que se ha constituido en un factor de atracción de inmigrantes a este territorio.

b. El conflicto en las regiones y zonas expulsoras de hogares.

c. Los desastres naturales, entre los que se cuenta las inundaciones del río Magdalena. Sin embargo durante la última década el factor determinante para el desplazamiento forzado y la consecuente llegada a Barrancabermeja está determinado por el conflicto armado que ha azotado a la mayoría de las regiones colombianas. Por lo que es preciso afirmar que en Barrancabermeja se observa claramente desde años atrás, la llegada de hogares que huyen de sus lugares de origen por motivo de violencia, por lo que la denominación Desplazamiento Forzado por Violencia, es un hecho real en el municipio.

En la presente investigación se realizaron 772 encuestas, en las que se caracteriza a la población en situación de desplazamiento objeto de análisis de este proyecto de la siguiente forma: La población atendida se encuentra en un rango de edad entre los 18 y 59 años, son pocas las personas mayores de 60 años, adultos mayores y menores de edad. La mayoría de la población tiene estudios de primaria, algunos con estudios de secundaria pero no culminan con el grado de bachiller, es poca la población que tiene estudios técnicos y ninguna persona atendida cuenta con estudios universitarios.

Las zonas de expulsión de víctimas de desplazamiento forzado a nivel nacional son por departamentos: Antioquia, Bolívar, Magdalena, Chocó, Cesar, Caquetá y Tolima. Y a nivel de Magdalena Medio los Departamentos de mayor expulsión son: Bolívar, Santander, Antioquia y Cesar, también se recepcionaron personas en menor proporción de los departamentos de Norte de Santander, Arauca, Magdalena, Boyacá, Sucre, Cundinamarca, Tolima, Atlántico, Meta, Caldas, Caquetá, Casanare, Cauca, Chocó, Córdoba, Guajira, Nariño, Putumayo y Valle del Cauca. Para analizar la vulnerabilidad de derechos de la población en situación de desplazamiento objeto de estudio, se realizó el estudio de las fases de la atención de acuerdo a la ley 387 de 1997, por el cual se establece la prevención, protección, atención y estabilización socioeconómica, como lo son: el Registro, la Atención de Urgencia, la Atención Humanitaria de Emergencia, la Estabilización Socioeconómica; y, adicionalmente, los derechos que se vuelven doblemente vulnerables, por la falta de garantía sistemática de las dificultades en la atención para garantizar el goce efectivo de derechos, son el Derecho a la Salud, Derecho a la Educación, Proyectos productivos, Derecho al Empleo, Derecho a la tierra y Derecho a la Vivienda. 
Tabla 1. Lugares de donde proviene la población en situación de desplazamiento

\begin{tabular}{|c|c|}
\hline $\begin{array}{c}\text { LUGAR DEL DESPLAZAMIENTO } \\
\text { DEPARTAMENTO }\end{array}$ & FRECUENCIA \\
\hline Total & 715 \\
\hline Bolívar & 237 \\
\hline Santander & 225 \\
\hline Antioquia & 164 \\
\hline Cesar & 20 \\
\hline Norte de Santander & 18 \\
\hline Arauca & 10 \\
\hline Magdalena & 9 \\
\hline Boyacá & 5 \\
\hline Sucre & 5 \\
\hline Cundinamarca & 4 \\
\hline Tolima & 3 \\
\hline Atlántico & 2 \\
\hline Meta & 2 \\
\hline Bajo Simacota & 1 \\
\hline Caldas & 1 \\
\hline Caquetá & 1 \\
\hline Casanare & 1 \\
\hline Cauca & 1 \\
\hline Choco & 1 \\
\hline Córdoba & 1 \\
\hline Guajira & 1 \\
\hline Nariño & 1 \\
\hline Putamayo & 1 \\
\hline Valle del Cauca & 1 \\
\hline
\end{tabular}

El Derecho a la salud se encuentra en primera medida garantizado al $86 \%$ de la población encuestada, el 14\% de la población no se encuentra inscrita en los servicios médicos, sin embargo, la vulnerabilidad de este derecho se presenta, con la prestación integral de los servicios, toda vez que las personas tienen la necesidad imperante de recibir atención psicológica de urgencia, médica y psicológica continuada, el embudo se dirige a la parte más baja, cuando de garantizar la prestación del servicio 
se trata, el 63\% recibió atención médica, y sólo el 47\% de la población inscrita recibió atención psicológica, menos de la mitad de la población continúa con el tratamiento de la superación de los hechos traumáticos causados por el desplazamiento forzado.

Barrancabermeja se debate entre una estrategia política militar de guerra, que desarrolla un proyecto de economía excluyente, autoritaria, marcada por la lógica del capital en un modelo agro exportador, minero extractivo y de enclave, de grandes negocios en la economía globalizada, y una apuesta a la paz, a la democracia, a la economía al servicio de la gente en armonía con la naturaleza impulsado por sectores de la ciudad de carácter civilista en los que aparece la iglesia, El Programa de Desarrollo y Paz del Magdalena Medio, las organizaciones de derechos humanos y sindicalistas entre otras.

Dos dinámicas con objetivos políticos antagónicos, que en su desarrollo las estructuras políticas, económicas, sociales de las autodefensas desmovilizadas utilizan una estratega integral de control territorial, que pasa por la coerción de los estamentos del poder público, la creación de alianzas hegemónicas y excluyentes entre ganaderos, narcotraficantes y el despliegue de redes de control político informales en áreas urbanas y rurales que al aislar y eliminar al contrario visible que era la izquierda armada del ELN y las FARC, el conflicto por el poder y el control de la economía lícita e ilícita toma una nueva dinámica en esta fase de desarrollo en la perspectiva de la precomposición del poder en Barrancabermeja y el Magdalena Medio.

En ausencia de un enemigo armado presente y de la lógica de la confrontación armada, la recomposición del poder local y regional requiere legitimar un orden social autoritario y hegemónico en una ciudad que tiene un acumulado de movimiento social, obrero y popular; recurre al uso indiscriminado de la violencia para someter y captar a las instituciones, organizaciones sociales, imponer conductas y patrones de convivencia, apropiar recursos y finanzas públicas y eliminar a quienes representen propuestas contrarias a las promovidas por la ultraderecha, en la que la población civil corre riesgos y queda expuesta a la ocurrencia de homicidios selectivos y configuración múltiple, enfrentamientos armados con interposición de población civil, destrucción de bienes civiles y el desplazamiento forzado.

En este marco de ideas el año 2006 inició con la desmovilización del Bloque Central Bolívar, que generó, expectativa en los pobladores toda vez que este actor armado ilegal se erigía como protagonista importante de la crisis humanitaria y de Derechos Humanos que padecía Barrancabermeja y la región del Magdalena Medio. Según datos de Acción Social, los motivos de desplazamiento se generaban desde la sindicación de pertenecer a organizaciones sociales defensoras de Derechos Humanos, sindicatos, organizaciones políticas de izquierda; hasta las desavenencias al interior de las autodefensas, hoy desmovilizadas, y el incumplimiento de cuotas reglamentarias en dinero de personas que utilizan el sistema de crédito fácil y no podían cancelar sus deudas en los plazos fijados. De igual manera un significativo número de jóvenes tuvieron que abandonar la ciudad por la presión de supuestos 
grupos de limpieza social quienes los sindican de ser drogadictos, ladrones o, simplemente por no corresponder a sus propósitos delincuenciales.

El desplazamiento forzado se constituye en una de las problemáticas sociales que requiere de grandes esfuerzos y exige asumir un compromiso por parte de la investigación a través de la Universidad y la sociedad civil en general. El desplazamiento interno en Colombia ha adquirido dimensiones preocupantes debido al recrudecimiento del conflicto armado en el país. Como testimonio de ello se encuentran las múltiples investigaciones y conocimientos producidos sobre el tema, los cuales se han presentado como descripciones de la problemática y constituyen un avance significativo. Pero, sin duda, se requiere de investigaciones bajo otros paradigmas que permitan comprender una dimensión más cercana al sentido real del problema.

Tal y como lo encuentra la investigación en lo referente a la categoría de efectos psicosociales, se encontró en los documentos analizados una significativa información, cuyos planteamientos centrales se dirigen a mostrar las graves consecuencias del desplazamiento y la necesidad de un abordaje interdisciplinario del fenómeno. Se evidencia, además, la urgencia que demanda la población desplazada por encontrar un nuevo rumbo para su vida y la de sus familias, por minimizar ese sentimiento de inseguridad desatado por la ausencia de futuro y por comenzar a generar estrategias a partir de las cuales las propias víctimas reconstruyan su tejido social, encuentren y construyan perspectivas de vida alcanzables con un claro sentido ético.

Las causas de desplazamiento en su orden de mayor relevancia son: amenazas generales y específicas, ataques indiscriminados, enfrentamientos armados, masacres y toma de población. Otras causas más específicas: desaparición forzada de un familiar, toma de población por parte de la guerrilla, toma de población por parte de los paramilitares, fumigaciones con glifosato, homicidio de familiar, homicidios de vecinos, miedo y reclutamiento de menores.

Para analizar la vulnerabilidad de derechos de la población en situación de desplazamiento objeto de estudio, se realizó el estudio de las fases de la atención de acuerdo a la ley 387 de 1997, por la cual se establece la prevención, protección, atención y estabilización socioeconómica, como lo son: el registro, la Atención de Urgencia, la Atención Humanitaria de Emergencia, la Estabilización Socioeconómica; y adicionalmente a ello, los derechos que se vuelven doblemente vulnerables, por la falta de garantía sistemática de las dificultades en la atención para garantizar el goce efectivo de derechos, son el Derecho a la Salud, Derecho a la Educación, Proyectos productivos, Derecho al Empleo, Derecho a la tierra y Derecho a la Vivienda. Para el registro de la población en situación de desplazamiento las personas acuden generalmente a la Personería Municipal de Barrancabermeja o al funcionario de la misma entidad que presta sus servicios en la Unidad de Atención y Orientación para población en Situación de Desplazamiento -UAO. Y también acuden en menor proporción a la Defensoría del Pueblo, a la Procuraduría no se acude porque esta entidad funciona como órgano de control para garantizar el cumplimiento de las 
fases de la atención de las diferentes instituciones vinculadas al Sistema Nacional de Atención Integral a Población Desplazada.

Las personas que declaran sobre su desplazamiento, han sufrido o han sido víctimas del mismo delito por actores, causas, en tiempos y lugares diferentes, no solamente de uno sino de dos y tres desplazamientos. Las personas que fueron víctimas varias veces, solamente declaran un solo hecho y las entidades del ministerio público no registran la magnitud de los sucesos, o la frecuencia con que le ocurre a la población que ha sido registrada, genera una vulnerabilidad en el derecho a la justicia, porque los anteriores hechos quedan totalmente en la impunidad.

El registro no se genera de forma automática con sólo declarar, por parte de Acción Social corresponde la valoración de la declaración y notificar a la persona en situación de desplazamiento la resolución de inclusión o no en el mismo. De la población declarante el $37 \%$ no fue incluida en el registro inmediatamente, el $63 \%$ fue incluido con solo la declaración, el $95 \%$ de la población no incluida inicialmente con la declaración, fue incluida con el agotamiento de la vía gubernativa, es decir, con interponer los recursos de reposición y en subsidio de apelación, el $5 \%$ de esa población fue incluida con los mecanismos de exigibilidad de derechos fundamentales, derechos de petición y acciones de tutelas. Las personas que han tenido más de dos desplazamientos, se les pregunta si han sido ofrecidas las opciones de reubicación o retorno, pero en pocos casos, casi que excepcionalmente se le orienta a la víctima declarante que puede optar por estas opciones de vida.

La encuesta contempló la opciones de retorno, reubicación o reasentamiento, a lo que las personas responden preferir ser reasentadas, quedarse en el lugar donde lo recibieron y declaró su desplazamiento para continuar con su vida, otro número considerable aceptaría la opción de hacer parte de procesos de reubicación, sin embargo la opción que menos se prefiere por falta de garantías de seguridad y de no repetición de los hechos que ocasionaron el desplazamiento forzado es la de retornar o volver a las tierras y lugares abandonados por la violencia.

Barrancabermeja ha desarrollado a nivel interinstitucional y coordinado desde la Alcaldía Municipal, específicamente de la Unidad de Atención y Orientación UAO, para garantizar el proceso de Atención de Urgencia, una ruta que permite a las personas garantizar sus necesidades básicas mientras se desarrolla el procedimiento de inclusión en el RUPD. El convenio suscrito entre la Alcaldía Municipal y el Servicio Jesuita a Refugiados garantiza el alojamiento y la alimentación de las familias en situación de desplazamiento, allí se le brinda atención médica y psicológica con la articulación de los programas del ICBF y la Secretaría de Salud. La mitad de la población encuestada aproximadamente ha recibido ayuda inmediata, no se podría afirmar que la otra mitad, no la ha recibido por falta de ofrecimiento institucional, dado que los tiempos de declaración y de ocurrencia del delito de desplazamiento forzado pueden variar, y consecuencia de ello, lo innecesario de brindar una atención de urgencia, por no encontrarse la persona en inminente necesidad o extrema urgencia. 
De la población que afirmó haber recibido ayuda inmediata, el $40 \%$ aproximadamente, dijo haber recibido alojamiento transitorio en casas, albergues o dinero para el alojamiento, la variación de estas opciones obedece a que con el pasar de los años varían las estrategias de atención por parte de las instituciones del SNAIPD para solucionar las situaciones de urgencias de la población en situación de desplazamiento. Más del $70 \%$ de la población que recibió alojamiento transitorio, fue dotada con los kits de aseo, cocina, habitad y alimentación.

La Coordinación interinstitucional se fractura en la fase de inclusión en el RUPD, toda vez que Acción Social, dilata los términos de valoración de la declaración y, a su vez, de la expedición de la resolución de inclusión o no inclusión, las personas que son atendidas en el Albergue tienen que permanecer un mes o más en espera de esta respuesta, y en ocasiones, corresponde al operador del mismo solicitar el desalojo voluntario de las familias, que en esas condiciones desbordan la capacidad presupuestal de la Alcaldía Municipal para su manutención y sostenimiento durante varios meses. Las personas en situación de desplazamiento que se encuentran en el Albergue deben desplazarse a las oficinas del Ministerio Público (cuando no son desplazamientos masivos), y a las instalaciones de la UAO para cubrir la ruta de inclusión en el Registro y para la Atención Humanitaria de Emergencia, esto expone a las personas a un mayor riesgo de seguridad y las distancias son considerables para acudir a dichas instituciones.

El $78 \%$ de la población encuestada recibió la atención humanitaria de emergencia, no se especifica en este análisis si fue en especie o en dinero, Acción Social reporta, para el tema del pronunciamiento de la Corte Constitucional, que sostuvo en la sentencia T-285 de 2008, que la prórroga de la atención humanitaria de emergencia, debe realizarse hasta que el afectado esté en condiciones de asumir su auto sostenimiento, que se han visto colapsados los recursos de esta fase con las constantes solicitudes de prórroga de la atención, sin embargo, el análisis reporta que solamente el 7\% de la población ha recibido la prórroga de la atención humanitaria de emergencia. La entrega de la Atención Humanitaria de Emergencia se ha convertido en una de las prioridades de Acción Social en Barrancabermeja, las personas han reclamado la Atención Humanitaria de Emergencia, pero esto no obedece a la atención sistemática, coordinada, planeada y progresiva para que las personas garanticen durante el tiempo estipulado por la ley sus necesidades básicas como alimentación y alojamiento, puesto que, se hacen jornadas masivas de asignación de la Atención Humanitaria de Emergencia, dónde a las personas les entregan un monto (diferenciado por tipos: A, B o C), y aparece en el sistema que se le ha garantizado esta fase de la atención, razón por la cual, las personas recurren posteriormente a la solicitud de la prórroga de la Atención Humanitaria de emergencia.

El 10\% de la población indagada responde haber recibido dinero o implementos para desarrollar su propio proyecto productivo. Ha sido este recurso para proyectos de emprendimiento, palma, ganadería, pollos, o actividades informales como la elaboración artesanal de productos para la venta, sandalias, correas, bolsos, muñecos, 
o el surtimiento de mercancía para la reventa en tiendas, almacenes y en sus casas. Existe una ruptura entre la coordinación de las instituciones del SNAIPD local, y de este a su vez con el departamental y nacional para el cumplimiento de la atención de esta fase, y que por consiguiente todo el peso de la atención a la población en situación de desplazamiento se devuelve a las primeras fases de atención convirtiéndose en una barrera para la superación del estado de cosas inconstitucional.

Los mecanismos, rutas y procedimientos creados por Acción Social solo obedecen a su propia dinámica interna con la entrega de Plan Semilla o Proyecto Productivo, en el municipio se ha avanzado en esta fase por los esfuerzos institucionales del SNAIPD local en garantizar de forma individual cada uno de los derechos de la población en situación de desplazamiento de acuerdo a su competencia. El 2\% de las personas que han implementado algún proyecto productivo ha buscado la forma de adquirir un crédito para hacer crecer el proyecto o el negocio, sin dar cuenta que los mismos prosperen o fracasen.

Los empleos otorgados, han sido en la zona urbana, cosa que nos permite indicar, que el proceso de adaptabilidad de las personas es casi nulo, debido a que las personas no cuentan con el estudio y la experticia de los empleos que requieren para trabajar en la zona urbana. El empleo, se ha considerado una de las principales garantías constitucionales para poder decir que se establece socioeconómicamente a la persona en situación de desplazamiento. Sin embargo, la Alcaldía Municipal ha generado políticas públicas para contrarrestar el tema de desempleo en la población en situación de desplazamiento, pero que por la gravedad de la situación se han convertido en medidas incipientes, insistiendo, en que son en vano los alcances municipales toda vez que este, no ha contado con el respaldo del nivel nacional y departamental.

Los empleos otorgados a la población en situación de desplazamiento en la zona urbana, obedece a subempleos, o a empleos que no requieren un conocimiento técnico o profesional, que pueden en caso ser empleos informales, sin las garantías y prestaciones sociales que denota la empleabilidad en un manejo de la política pública de empleo para la población en situación de desplazamiento, y poniendo en evidencia, que se vulnera el derecho al trabajo de la población en situación víctima de este delito. Hay un total de 1.856 hectáreas abandonadas por el delito del desplazamiento forzado del número de personas encuestadas. Que el 49\% de la población dice ser propietaria de las tierras, el $30 \%$ tenedor y el $21 \%$ poseedor, el vínculo mayor con el inmueble es el propietario con justo título. Se observa, que hay una distribución equitativa de la tierra, es decir, la mayoría de los propietarios, son dueños de pequeñas extensiones de tierra, de parcelas con poca magnitud de terreno, pero que sumadas, dan cuenta de la gran cantidad de población rural víctima del desplazamiento forzado. 
Tabla 2. Frecuencia de los empleos realizados por la población en situación de desplazamiento en la zona urbana

\begin{tabular}{|c|c|}
\hline EN QUE REALIZÓ EL EMPLEO & FRECUENCIA \\
\hline Albañilería & 2 \\
\hline Cosiendo forros para computador & 2 \\
\hline Empleada doméstica & 2 \\
\hline Jardinería & 2 \\
\hline Impulsadora & 2 \\
\hline Lavando y planchando & 2 \\
\hline Yambal & 2 \\
\hline Total & 14 \\
\hline
\end{tabular}

Las personas en situación de desplazamiento han perdido y dejado abandonadas las tierras o bienes inmuebles en el área rural y urbana, pero esta situación ha quedado de lado como componente de reparación toda vez, que el Desplazamiento Forzado se declara para efectos meramente administrativos, pero no para la protección del derecho que involucra el área penal y policivo. El 89\% de la población no ha solicitado subsidio de vivienda, iniciando con el primer paso de la ruta que garantizaría este derecho. $11 \%$ de los encuestados dice haber solicitado el subsidio. Del 11\% que solicitó el subsidio el $67 \%$ ha recibido el subsidio de vivienda, el 33\% de esa población no lo recibe. Hay que anotar que el contexto económico en que vive la ciudad de Barrancabermeja, por el auge petrolero y la industria, ha generado que los costos en el nivel de vida se haya aumentando, no queriendo decir que sea directamente proporcional con el desarrollo social, sin embargo, ha creado un alza incontrolable de los precios de las viviendas que imposibilita a la administración municipal demostrar los esfuerzos realizados en este tema.

\section{Lectura de resultados}

La lectura de los resultados encontrados, deja observar en esta investigación, la situación sobre el tema de desplazamiento forzado en Colombia, a partir de los múltiples argumentos descriptivos obtenidos a través de las fuentes documentales analizadas. Si bien se constituyó en un esfuerzo importante, es preciso darle continuidad, bien sea a partir de la comprensión e interpretación de cada una de las categorías de análisis establecidas o bien comenzando con otras nuevas, bajo el entendido que Barrancabermeja históricamente se he configurado como territorio de multiculturalidad, de inmigraciones y emigraciones. 
La lectura permite revisar los siguientes resultados: se realizaron 772 encuestas, en las que se caracteriza a la población en situación de desplazamiento objeto de análisis de este proyecto de la siguiente forma: La población atendida se encuentra en un rango de edad entre los 18 y 59 años, son pocas las personas mayores de 60 años, adultos mayores y menores de edad. La mayoría de la población solamente tiene estudios de primaria, algunos con estudios de secundaria pero no culminan con el grado de bachiller, es poca la población que tiene estudios técnicos y ninguna persona atendida cuenta con estudios universitarios. Las zonas de expulsión de víctimas de desplazamiento forzado a nivel nacional son por departamentos: Antioquia, Bolívar, Magdalena, Chocó, Cesar, Caquetá y Tolima. Y a nivel de Magdalena medio los Departamentos de mayor expulsión son: Bolívar, Santander, Antioquia y Cesar, también se recepcionaron personas en menor proporción de los departamentos de Norte de Santander, Arauca, Magdalena, Boyacá, Sucre, Cundinamarca, Tolima, atlántico, meta, Caldas, Caquetá, Casanare, Cauca, Chocó, Córdoba, Guajira, Nariño, Putumayo y Valle del Cauca.

Los municipios de mayor expulsión por Departamento, vienen siendo las cabeceras municipales como puertos fluviales, pero no son expulsoras en mayor ni menor porcentaje las capitales de cada departamento. Así, en el departamento de Bolívar, son expulsores en mayor proporción, Cantagallo y San Pablo, pertenecientes al Sur de Bolívar, en Santander Barrancabermeja y Simacota, y en el Departamento de Antioquia Yondó y Puerto Berrio. Las causas de desplazamientos en su orden de mayor relevancia son: Amenazas, generales y específicas, ataques indiscriminados, enfrentamientos armados, masacres y toma de población. Otras causas más específicas: Desaparición forzada de un familiar, toma de población por parte de la guerrilla, toma de población por parte de los paramilitares, fumigaciones con glifosato, homicidio de familiar, homicidios de vecinos, miedo y reclutamiento a menores.

Hay un total de 1.856 hectáreas abandonadas por el delito del desplazamiento forzado del número de personas encuestadas. El 49\% de la población dice ser propietaria de las tierras, el $30 \%$ tenedor y el $21 \%$ poseedor, siendo el vínculo mayor con el inmueble el propietario con justo título. Se observa, que hay una distribución equitativa de la tierra, es decir, la mayoría de los propietarios, son dueños de pequeñas extensiones de tierra, de parcelas con poca magnitud de terreno, pero que sumadas, dan cuenta de la gran cantidad de población rural víctima del desplazamiento forzado. Las personas en situación de desplazamiento han perdido y dejado abandonadas las tierras o bienes inmuebles en el área rural y urbana, pero esta situación ha quedado de lado como componente de reparación toda vez, que el Desplazamiento Forzado se declara para efectos meramente administrativos, pero no para la protección del derecho que involucra el área penal y policivo.

El $89 \%$ de la población no ha solicitado subsidio de vivienda, iniciando con el primer paso de la ruta que garantizaría este derecho, 11\% de los encuestados dice haber solicitado el subsidio. Del $11 \%$ que solicitó el subsidio el $67 \%$ ha recibido el 
subsidio de vivienda, el 33\% de esa población no lo recibe. Hay que anotar que el contexto económico en que vive la ciudad de Barrancabermeja, por el auge petrolero y la industria, ha generado que los costos en el nivel de vida se haya aumentando, no queriendo decir que sea directamente proporcional con el desarrollo social, sin embargo, ha creado un alza incontrolable de los precios de las viviendas, imposibilitando a la administración municipal demostrar los esfuerzos realizados en este tema.

\section{CONCLUSIONES}

Las zonas de expulsión de víctimas de desplazamiento forzado a nivel nacional son por departamentos: Antioquia, Bolívar, Magdalena, Chocó, Cesar, Caquetá y Tolima. Y a nivel de Magdalena medio los Departamentos de mayor expulsión son: Bolívar, Santander, Antioquia y Cesar, también se recepcionaron personas en menor proporción de los departamentos de Norte de Santander, Arauca, Magdalena, Boyacá, Sucre, Cundinamarca, Tolima, atlántico, meta, Caldas, Caquetá, Casanare, Cauca, Chocó, Córdoba, Guajira, Nariño, Putumayo y Valle del Cauca. Los municipios de mayor expulsión por Departamento, vienen siendo las cabeceras municipales como puertos fluviales, pero no son expulsoras en mayor ni menor porcentaje las capitales de cada departamento. Así, en el departamento de Bolívar, son expulsores en mayor proporción, Cantagallo y San Pablo, pertenecientes al Sur de Bolívar, en Santander Barrancabermeja y Simacota, y en el Departamento de Antioquia Yondó y Puerto Berrío.

El municipio de Barrancabermeja ha venido haciendo esfuerzos institucionales desde el año 2006 para implementar políticas públicas de atención a la Población en situación de desplazamiento. No obstante, se presentan dificultades en la coordinación de los comités encargados de llevar adelante dichas políticas. Dentro de las dificultades más frecuentes se encuentran, el fortalecimiento de los canales de comunicación entre los comités y entes encargados de brindar asistencia a la población, la falta de organización de información recogida de dicha población, la demora para tomar decisiones fundamentales que impactan a la población en situación de desplazamiento y demás.

El problema de la coordinación en la asistencia de la PSD no es un problema menor, ya que finalmente se sabe que sin una política pública seria, será muy difícil que se puedan superar las condiciones a las que este fenómeno somete a sus afectados. Además se ha evidenciado que existen contradicciones desde la esfera de lo político en la asistencia y tratamiento a esta población desde la implementación de las políticas públicas, para contrarrestar estos efectos desde el ámbito nacional se han realizado esfuerzos para convertir las políticas de gobierno en políticas de Estado, y así poder garantizar la continuidad y perfeccionamiento de las políticas ideadas para combatir y superar este fenómeno gradualmente, con eficacia y celeridad. 
Igualmente se evidencio, que los entes involucrados en el afrontamiento del fenómeno de desplazamiento forzado, a pesar del interés demostrado, tienen grandes dificultades en el tema de la caracterización de la PSD, lo que trae retrasos en la capacidad de respuesta al fenómeno, esto en parte sigue siendo resultado de la inexistencia de un enfoque común para el tratamiento del fenómeno y la escasa coordinación del orden nacional-municipal en esta problemática, falta control desde lo nacional y un compromiso más sistemático y decidido desde lo municipal, para cumplir con los compromisos de atención integral a la PSD en el municipio de Barrancabermeja. Aunque hay que apuntar, que desde el año 2009 se vienen realizando sendos esfuerzos para superar estas condiciones que dificultan la aceleración del proceso de superación del fenómeno que ha padecido la región del magdalena medio con más intensidad desde hace por lo menos dos décadas.

\section{REFERENCIAS}

Archila, M. (1991). Cultura e identidad obrera. Bogotá, CINEP.

CODHES. (2000). Guerra del desplazamiento y pobreza. En CODHES Informa. Boletín de la Consultoría para los Derechos Humanos y Desplazamientos a Marchas Forzadas. 30 (agosto). CODHES. Bogotá.

Conferencia Episcopal de Colombia. (1995). Boletín Trimestral sobre el Desplazamiento Forzado en Colombia. 8 (septiembre-diciembre). Editorial Kimpres. Bogotá.

Contreras, Daniel, Olivares, Alejandro. Artículo de opinión: "Democracia en América Latina" en http://aolivares.blogspot.com/2005_08_01_aolivares_archive

Departamento Nacional de Planeación-DNP, Colombia. (1995). Programa Nacional de Atención Integral a la Población Desplazada por la Violencia. Documento Conpes 2804. Ministerio del Interior-Consejería Presidencial para los Derechos Humanos. Bogotá.

Diócesis de Barrancabermeja. (2001). La luz de esperanza, Barrancabermeja.

Falla, U., Chávez Y. y Molano, G. (2001). Desplazamiento forzado en Colombia: Estado del arte y una experiencia en la Unidad de Atención Integral a esta población. Informe final de la investigación. Universidad Colegio Mayor de Cundinamarca. Bogotá.

Franco, A. (1998). Los desplazamientos internos en Colombia. Una conceptualización política para el logro de soluciones a largo plazo. En Colombia Internacional. Publicación del Centro de Estudios Internacionales de la Universidad de los Andes. 42 (abril-junio): 5-27. Universidad de los Andes. Bogotá.

González, F. (1997). Aproximación a la configuración Política Colombiana, en Para Leer la Política, Tomo I, CINEP, Santafé de Bogotá. 
Guillen, F. (1986). La Regeneración” Primer Frente Nacional, Carlos Valencia Editores, Bogotá. Págs. 15-70 los partidos políticos.

Grupo Organizaciones de Apoyo a Desplazados. (2001). Éxodo. Boletín de Desplazamiento Interno en Colombia. Editorial GAD. Bogotá.

López, O. (2000). Investigación y trabajo psicosocial con familias víctimas de la guerra. Revista Colombiana de Trabajo Social. Consejo Nacional para la Educación en Trabajo Social. Serie 14. CONETS. Cali.

Ministerio de Salud-Fundación Educativa Amor (s.f.). Presentación propuesta de adaptación psicosocial a familias desplazadas de campo por la violencia. CINEP. Bogotá. Naciones Unidas-Defensoría del Pueblo, Colombia. Principios rectores de los desplazamientos internos. Naciones Unidas-Defensoría del Pueblo. Bogotá.

Organización Panamericana de la Salud-CODHES-UNICEF. (1999). Un país que huye. Desplazamiento y violencia: una nación fragmentada. Ed. Guadalupe. Bogotá.

Osorio, F. (1993). La violencia del silencio: desplazados del campo a la ciudad. CODHES -Pontificia Universidad Javeriana. Bogotá.

Pastrana, A, López, C y Solimano, A. (s.f.). Ensayo sobre paz y desarrollo y otros. Tercer Mundo Editores. Bogotá.

Pérez, D. (1995). El desplazamiento forzado en Colombia: ruptura del tejido social y el proyecto vital». En Su Defensor. Periódico de la Defensoría del Pueblo para la divulgación de los derechos humanos. 21. Defensoría del Pueblo. Bogotá.

PNUD. (2004). "El desarrollo de la democracia en América Latina". En la Democracia en América Latina. Hacia una democracia de ciudadanas y ciudadanos, PNUD.

Vargas, A. (1998). Las Fuerzas Armadas en el Conflicto Colombiano. Antecedentes y perspectivas, Intermedio Editores, Bogotá. Capítulo 2. 